\title{
CONTINUUM-CHAINABLE CONTINUUM WHICH CAN NOT BE MAPPED ONTO AN ARCWISE CONNECTED CONTINUUM BY A MONOTONE EPSILON MAPPING
}

\author{
Pavel Pyrih, Benjamin Vejnar and Luis Miguel García Velázquez \\ Charles University in Prague, Czech Republic and Universidad Nacional \\ Autonoma de Mexico, Mexico
}

\begin{abstract}
A continuum is called continuum-chainable provided for any pair of points and positive epsilon there exists a finite weak chain of subcontinua of diameter less than epsilon starting at one point and ending in the other. We present an example of a continuum which is continuum-chainable and which can not be mapped onto an arcwise connected continuum by a monotone epsilon mapping. This answers a question posed by W. J. Charatonik.
\end{abstract}

\section{INTRODUCTION}

A continuum is a compact connected metrizable space. A continuum $X$ is called continuum-chainable provided for any pair of points $x, y \in X$ and $\varepsilon>0$ there exists $n \in \mathbb{N}$ and continua $K_{1}, \ldots, K_{n}$ of diameter at most $\varepsilon$ such that $K_{i} \cap K_{i+1} \neq \emptyset$ for $i<n, x \in K_{1}$ and $y \in K_{n}$. A mapping $f: X \rightarrow Y$ is called monotone if it is continuous and $f^{-1}(y)$ is connected for every $y \in Y$. A continuous mapping $f$ of a metric space $X$ to a space $Y$ is called an $\varepsilon$-mapping if $f^{-1}(y)$ is either empty or of diameter less than $\varepsilon$.

We denote by $\mathcal{C}$ the class containing any continuum $X$, such that for every $\varepsilon>0$ there exists an arcwise connected continuum $X_{\varepsilon}$ and a surjective monotone $\varepsilon$-mapping $f_{\varepsilon}: X \rightarrow X_{\varepsilon}$. We note that the property of being an element of the class $\mathcal{C}$ is a topological property.

It can be easily shown that any continuum from the class $\mathcal{C}$ is continuumchainable. There was a question asked by W. J. Charatonik during the

2010 Mathematics Subject Classification. 54G20, 54F50.

Key words and phrases. Continuum, continuum-chainable, monotone mapping, arcwise connected.

The work was supported by the grant SVV-2012-265316. 
Continuum Theory Prague 2011, Open Problem Workshop, whether the converse is true. This question is related to the notion of a representation space (see [ChIP] for some details). Roughly speaking it can be reformulated as follows. Is it true that the 'closure' of the class of all arcwise connected continua in the representation space coincides with the class $\mathcal{C}$ ?

We answer the question negatively. That is, we give an example of a continuum-chainable continuum which is not an element of the class $\mathcal{C}$.

Let $d$ be a metric for a space $X, x$ a point in $X, \delta$ a positive number and $A$ a subset of $X$. We denote by $B_{\delta}(x)$ the set $\{y \in X: d(x, y)<\delta\}$ and by $N_{\delta}(A)$ the set $\{y \in X: d(y, a)<\delta$ for some $a \in A\}$.

A proof of the following lemma can be found in [N, Exercise 2.33].

Lemma 1.1. Let $\Psi$ be an $\varepsilon$-mapping of a continuum $X$ onto $Y$. Then there exists $\nu>0$ such that if $A \subseteq Y$ and $A \subset B_{\nu}(y)$, then $\operatorname{diam}\left(\Psi^{-1}(A)\right)<\varepsilon$.

\section{EXAMPLE}

We define in $\mathbb{R}^{3}$ the $\sin \frac{1}{x}$-continuum $S=A \cup L$ where $A=\{0\} \times[-1,1] \times$ $\{0\}$ and $\left.L=\left\{\left(x, \sin \frac{1}{x}, 0\right): 0<x \leq 1\right]\right\}$. We consider a sequence of arcs

$$
D_{n}=\left\{\left(x, \sin \frac{1}{x}, 2^{-n} x\right): \frac{1}{\pi n} \leq x \leq 1\right\}
$$

which approximate the continuum $S$. We define a ray

$$
C_{n}=\left\{\left(x, 2^{-n} \sin \frac{1}{x}, 2^{-n} x\right): 0<x \leq \frac{1}{\pi n}\right\}
$$

for any $n \in \mathbb{N}$. We put $L_{n}=C_{n} \cup D_{n}$ which is a ray first approximating a compact subset of $L$ and then oscilating near the arc $\{0\} \times\left[-2^{-n}, 2^{-n}\right] \times\{0\}$. We denote by $Q$ the $\operatorname{arc}\{(1, \sin 1)\} \times[0,1]$ and we put

$$
P=S \cup Q \cup L_{1} \cup L_{2} \cup \ldots
$$

Moreover we denote by $I_{n}$ the subarc of $A$ which joins the points $\left(0,-2^{-n}, 0\right)$ and $\left(0,2^{-n}, 0\right)$. Hence $I_{n}$ is a set of all cluster points of the ray $L_{n}$.

\section{Claim 1. Continuum $P$ is continuum-chainable.}

Proof. Suppose that $x, y \in P$ and $\varepsilon>0$. We note that $P$ has two arc components. If both points $x$ and $y$ are in the same arc component, then we are easily done. Thus we may suppose that they are in different arc components. We can find a natural number $n$ such that $2^{-n}<\frac{\varepsilon}{2}$. There is a chain of continua in $A \cup L_{n}$ of diameter at most $\varepsilon$ joining the two arc components. Now, we can prolong this chain to obtain a chain joining the points $x$ and $y$.

We remark, that the continuum $P$ can be mapped onto an arcwise connected continuum by a monotone $\varepsilon$-mapping for every $\varepsilon>0$. It is enough to shrink the arc $\{0\} \times\left[-\frac{\varepsilon}{3}, \frac{\varepsilon}{3}\right] \times\{0\}$ into a single point and to leave the rest. 


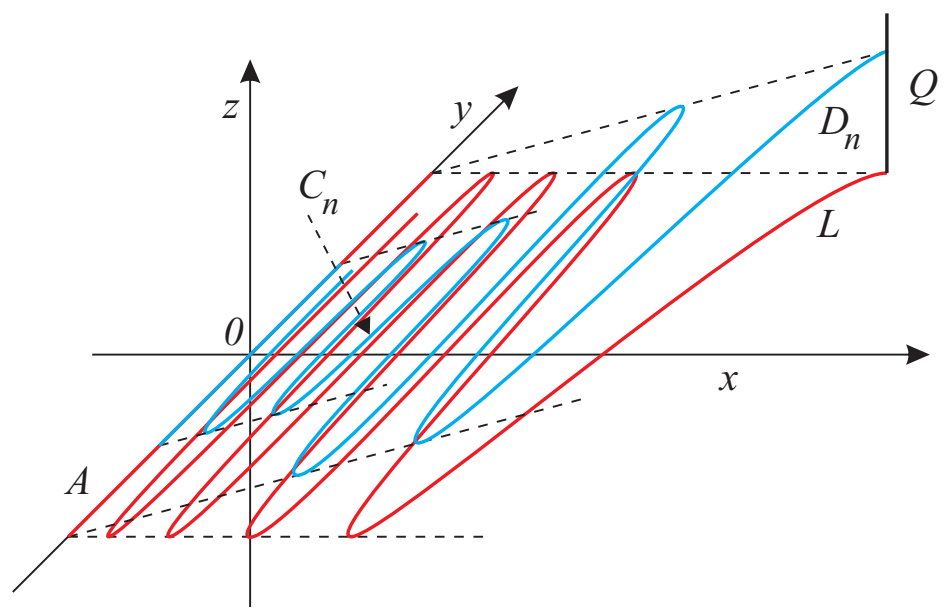

Figure 1. Continuum P.

Claim 2. If $E$ is a subcontinuum of $P$ which intersects $A$ and $P \backslash A$, then $(0,0,0) \in E$.

Proof. Obvious.

Now, we can use the feature of $P$ from Claim 2 to construct a continuum with a lot of problematic points. Let $f: C \rightarrow[-1,1]$ be a continuous mapping of the Cantor set $C$ onto the interval $[-1,1]$ (we may suppose that $f$ is for example restriction of the classical Cantor function composed with an affine function). We consider the product $P \times C$ and we define a mapping

$$
\varphi: A \times C \rightarrow[-2,2], \quad \varphi(0, a, 0, c)=a+f(c)
$$

for $a \in[-1,1]$ and $c \in C$. We define the desired space $X$ as an attaching space $(P \times C) \cup_{\varphi}[-2,2]$, that is a quotient of the disjoint union $(P \times C) \cup[-2,2]$ using the upper semicontinuous decomposition

$$
\left\{\{x\} \cup \varphi^{-1}(x): x \in[-2,2]\right\} \cup\{\{p\}: p \in(P \backslash A) \times C\} .
$$

Thus we obtain a book with uncountable many pages, where pages are copies of the continuum $P$. They are not binded in the ordinary way, but using a shift. Let us denote by $q$ the quotient mapping of $(P \times C) \cup[-2,2]$ onto $X$. We denote by $P_{c}$ the page $q(P \times\{c\})$ and by $H$ the shelfback $q([-2,2])$ of the book $B$.

We show that the book $B$ is a continuum chainable continuum which can not be mapped onto an arcwise connected continuum by a monotone $\varepsilon$-mapping for $\varepsilon>0$ small enough.

Claim 3. Continuum B is continuum-chainable. 


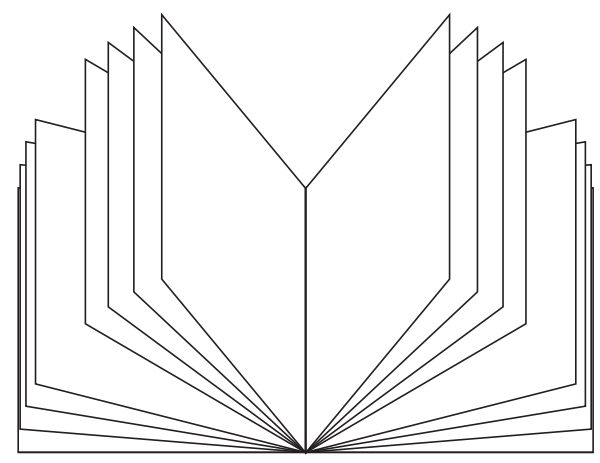

Figure 2. An ordinary book.

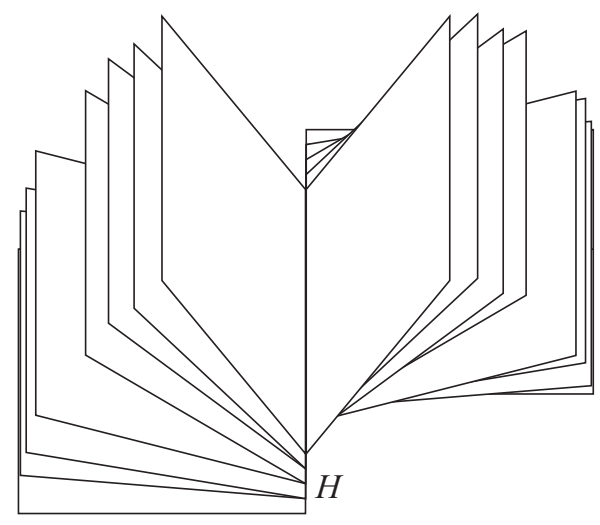

Figure 3. Continuum $B$.

Proof. It follows easily by the definition of continuum-chainability using Claim 1 and the construction of the continuum $B$.

Claim 4. The components of $B \backslash H$ are the elements of $\left\{P_{c} \backslash H: c \in C\right\}$.

Proof. The components of the space $(P \times C) \backslash(A \times C)$ are the sets of the form $(P \backslash A) \times\{c\}$, where $c \in C$. Our claim follows from the fact that $q$ restricted to $(P \backslash A) \times C$ is a homeomorphism.

Let $d$ be a metric for the book $B$. We consider $\varepsilon>0$ which is smaller than the minimum distance from points of $q(Q \times C)$ to points of $H$ and which is also smaller than the diameter of $q([-1,1])$.

Claim 5. Let $E$ be a subcontinuum of $B$ such that $\operatorname{diam}(E)<\varepsilon$. Let $c \in C$ and $n \in \mathbb{N}$. If $E$ intersects $H$ as well as $q\left(L_{n} \times\{c\}\right)$ then $q\left(I_{n} \times\{c\}\right) \subset E$. 
Proof. It is clear that $E \cap q(Q \times C)=\emptyset$ since $\operatorname{diam}(E)<\varepsilon$. Let $b \in q\left(L_{n} \times\{c\}\right) \cap E$ and let $D$ be the component of $q\left(L_{n} \times\{c\}\right) \backslash\{b\}$ which does not intersect $q(Q \times C)$. We show that $D \subset E$. Suppose the opposite and take $x \in D \backslash E$. Since $E$ is a continuum it should be contained in the component of $B \backslash(\{x\} \cup q(Q \times C))$ containing $b$. Then $E \cap H=\emptyset$, which is a contradition. Therefore $D \subset E$. Since $q\left(I_{n} \times\{c\}\right)$ is a subset of the closure of $D$ we get that $q\left(I_{n} \times\{c\}\right) \subset E$, because $E$ is closed.

ClAIM 6. Let $\Psi$ be a monotone $\varepsilon$-mapping of $B$ onto an arcwise connected continuum $Y$. Then for every $c \in C$ there exists $\delta>0$ such that the restriction of the mapping $\Psi$ to the set $q([f(c)-\delta, f(c)+\delta])$ is constant.

Proof. We fix any $c \in C$, a point $a \in P_{c} \backslash N_{\varepsilon}(H)$ and a point $b \in P_{c} \cap H$. We note that $\Psi(a) \neq \Psi(b)$ because $d(a, b) \geq \varepsilon$. Since $Y$ is arcwise connected, there exists a one-to-one map $\alpha:[0,1] \rightarrow Y$ such that $\alpha(0)=\Psi(a)$ and $\alpha(1)=\Psi(b)$.

Since $\Psi$ is an $\varepsilon$-mapping we get that $\alpha(0) \notin \Psi(H)$. Notice that $\alpha(1) \in$ $\Psi(H)$. Let $t_{0}=\inf \{t \in[0,1]: \alpha(t) \in \Psi(H)\}$. Since $\Psi(H)$ is compact, we have that $\alpha\left(t_{0}\right) \in \Psi(H)$. Therefore $t_{0}>0$. We denote $y=\alpha\left(t_{0}\right)$.

For $0<t \leq t_{0}$ we define $A_{t}=\Psi^{-1}(\alpha([0, t]))$. Since $\Psi$ is monotone $A_{t}$ is connected. For $t<t_{0}$ we have that $A_{t} \cap H=\emptyset$, hence $A_{t}$ is contained in the component of $B \backslash H$ that contains the point $a$. According to Claim 4 , this component is $P_{c} \backslash H$.

Since $\Psi$ is an $\varepsilon$-mapping we get by Lemma 1.1 that there is $\nu>0$ such that for any subcontinuum $A$ of $Y$ with $A \subset B_{\nu}(y)$ diameter of $\Psi^{-1}(A)$ is less than $\varepsilon$. We take $t_{1} \in\left(0, t_{0}\right)$ satisfying $\alpha\left(\left[t_{1}, t_{0}\right]\right) \subset B_{\nu}(y)$. We have that $B_{1}=\Psi^{-1}\left(\alpha\left(\left[t_{1}, t_{0}\right]\right)\right)$ is a subcontinuum of $B$ with diameter less than $\varepsilon$ which intersects $H$ and does not intersect $q(Q \times\{c\})$. We take $z_{1} \in \Psi^{-1}\left(\alpha\left(t_{1}\right)\right)$. Since $t_{1}<t_{0}$, we obtain that $z_{1} \in P_{c} \backslash H$. There is $n \in \mathbb{N}$ such that the ray $q\left(L_{n} \times\{c\}\right)$ contains the point $z_{1}$. By Claim 5 we obtain that $q\left(I_{n} \times\{c\}\right) \subset B_{1}$.

We take any sequence $\left(t_{k}: k \geq 2\right)$ of points in [0,1] whose limit is $t_{0}$ and such that $t_{1}<t_{2}<\ldots$. For each $k \geq 2$ we denote $B_{k}=\Psi^{-1}\left(\alpha\left(\left[t_{k}, t_{0}\right]\right)\right)$ and we fix a point $z_{k} \in \Psi^{-1}\left(\alpha\left(t_{k}\right)\right)$; since $\Psi$ is monotone, $B_{k}$ is a subcontinuum of $B$. For every $k \in \mathbb{N}$ the continuum $B_{k}$ intersects $H$ and $P_{c} \backslash H$, and has diameter less than $\varepsilon$. Since $\Psi$ is monotone we have that $\Psi^{-1}\left(\alpha\left(\left[t_{k}, t_{k+1}\right]\right)\right)$ is a subcontinuum of $A_{t_{k+1}}$, additionally it does not intersect $q(Q \times\{c\})$, because it is also a subcontinuum of $B_{1}$. Thus it should be contained in a component of $P_{c} \backslash(H \cup q(Q \times\{c\}))$. Since $z_{1} \in q\left(L_{n} \times\{c\}\right)$ we get that $z_{k} \in q\left(L_{n} \times\{c\}\right)$ for each $k \in \mathbb{N}$. Using Claim 5 we conclude that $q\left(I_{n} \times\{c\}\right) \subset B_{k}$.

Since $\Psi^{-1}(y)=\bigcap_{k=1}^{\infty} B_{k}$, we get that $\Psi^{-1}(y) \supset q\left(I_{n} \times\{c\}\right)=q([f(c)-$ $\delta, f(c)+\delta])$ for some $\delta>0$. Thus $\Psi$ is constant on the set $q([f(c)-\delta, f(c)+\delta])$. 
Claim 7. There does not exist a monotone $\varepsilon$-mapping from $B$ onto an arcwise connected continuum.

Proof. Suppose for the contrary that there is a monotone $\varepsilon$-mapping $\Psi$ of $B$ onto an arcwise connected continuum $Y$. By Claim 6 we get that for every $c \in C$ there is $\delta_{c}>0$ such that $\Psi$ is constant on the set $q\left(\left[f(c)-\delta_{c}, f(c)+\delta_{c}\right]\right)$. Since the set $q([-1,1])$ is connected we get that $\Psi$ is constant on $q([-1,1])$. By our choice of $\varepsilon$ we have that $\operatorname{diam}(q([-1,1]))>\varepsilon$ and thus we get a contradiction with the fact that $\Psi$ is an $\varepsilon$-mapping.

Problem 2.1. Give an internal characterization of the class of all continua that can be mapped onto arcwise connected continua by a monotone $\varepsilon$-mapping for every $\varepsilon>0$.

\section{REFERENCES}

[ChIP] W. J. Charatonik, M. Insall and J. R. Prajs, Connectedness of the representation space for continua, Topology Proc. 40 (2012), 331-336.

[N] S. B. Nadler Jr, Continuum theory. An introduction, Marcel Dekker, New York, 1992.

P. Pyrih

Faculty of Mathematics and Physics

Charles University in Prague

11800 Prague

Czech Republic

B. Vejnar

Faculty of Mathematics and Physics

Charles University in Prague

11800 Prague

Czech Republic

E-mail: vejnar@karlin.mff.cuni.cz

L. M. García Velázquez

Universidad Nacional Autónoma de México

Mexico City, D. F.

Mexico

E-mail: lmgarcia@matem. unam.mx

Received: 10.6.2012. 